\title{
Cultural differences between surgical and medical teams: is it time for comanagement?
}

Nathan Peiffer-Smadja ${ }^{1,2}$, Thomas Hanslik ${ }^{3}$, Pierre Mordant ${ }^{4}$, François-Xavier Lescure $^{1,2}$

1. Infectious Diseases Department, Bichat-Claude Bernard Hospital, AssistancePublique Hôpitaux de Paris, France.

2. Inserm, IAME, UMR 1137, Université Paris Diderot, Paris, France.

3. Internal Medicine Department, Université de Versailles Saint Quentin, Versailles, France.

4. Division of Thoracic and Vascular Surgey Department, Bichat-Claude Bernard Hospital, Assistance-Publique Hôpitaux de Paris, France.

Corresponding author:

Nathan Peiffer-Smadja

Infectious Diseases Department, Bichat-Claude Bernard Hospital, AssistancePublique Hôpitaux de Paris, France

Email: nathan.psmadja@gmail.com 


\section{Dear Editor,}

We have read with much interest the article of Charani and colleagues entitled "The Differences in Antibiotic Decision-making Between Acute Surgical and Acute Medical Teams: An Ethnographic Study of Culture and Team Dynamics". ${ }^{1}$ They demonstrate important differences in the functioning of medical and surgical teams, including a more individualistic approach, a more acute fear of blame and a shorter span of available time for the ward round in surgery.

In their article, they propose such solutions as adapting to surgeons' working hours or engaging in communication platforms used by surgeons. The associated editorial underlines the need for stewardship interventions tailored to the context of surgery. ${ }^{2}$

Such solutions are essential, however, most the causes put forward to account for these differences seem to us structural. Indeed, the fact that the surgeons have to share their time between the ward, clinics and the operating room, which forces them to do the round very quickly and makes it difficult to decide collectively, is not easily modifiable. Likewise, the "interventionist" side of surgery and consequently, "the impetus "to "do something"" is part and parcel of surgery.

We propose developing comanagement between surgeons and medical physicians as another solution. In this model, a physician trained in internal medicine takes care of chronic medical comorbidities and acute complications of the surgical patient, including infections and antibiotic prescribing. ${ }^{3}$

Comanagement is different from standard consultation where the surgeon retains sole authority and responsibility for identifying patients who would need a medical evaluation and can ignore the internist's recommendations. ${ }^{4}$ Comanagement is the result of a formal and negotiated agreement, ideally written down between the surgeon and the internist (sometimes called "hospitalist") to share the responsibility, authority and accountability for the care of surgical patients. The internist takes care of patients without a specific referral by the surgeon and is able to write out medical orders, communicate with the patient and other specialists including pharmacists.

As we are of the opinion that Charani and colleagues' findings are not limited to 
antibiotic prescribing and concern many medical decisions in surgical wards, comanagement seems a broader solution.

The practice of comanagement is gaining popularity, mainly in the USA. ${ }^{5}$ The only randomized controlled trial to date found that comanagement reduced the number of complications before hospital discharge. ${ }^{6}$ Other studies suggest that comanagement improves patient outcomes, including decreasing mortality, ${ }^{7,8}$ and reduced length of hospital stay. ${ }^{9}$ Moreover, surgeons and nurses sound more satisfied with comanagement than medical consultation and it could lead to reduced costs. ${ }^{6,10}$ To our knowledge, no study so far has addressed the impact of comanagement of surgical patients on antibiotic prescribing.

In France and the UK, comanagement is not widely used but we think that everybody would benefit from it, starting with surgeons who could spend more time in the operating room while of course participating in medical decisions. Antimicrobial stewardship teams would keep a crucial role advising internists working in surgical wards.

To meet this call, we think that a discussion at national levels should be started on comanagement of surgical patients between internists, surgeons and antimicrobial prescribing specialists. Such a proposal would need the support of public health specialists and political authorities to train more physicians in the medical management of surgical patients.

All authors confirm that they have no potential conflicts of interest to disclose 
References:

1. Charani E, Ahmad R, Rawson TM, Castro-Sanchèz E, Tarrant C, Holmes AH. The Differences in Antibiotic Decision-making Between Acute Surgical and Acute Medical Teams: An Ethnographic Study of Culture and Team Dynamics. Clin Infect Dis. November 2018. doi:10.1093/cid/ciy844

2. Szymczak JE. Are Surgeons Different? The Case for Bespoke Antimicrobial Stewardship. Clin Infect Dis. November 2018. doi:10.1093/cid/ciy847

3. Whinney C, Michota F. Surgical comanagement: A natural evolution of hospitalist practice. Journal of Hospital Medicine. 2008;3(5):394-397. doi:10.1002/jhm.359

4. Klein LE, Levine DM, Moore RD, Kirby SM. The preoperative consultation. Response to internists' recommendations. Arch Intern Med. 1983;143(4):743-744.

5. Sharma G, Kuo Y-F, Freeman J, Zhang DD, Goodwin JS. Comanagement of hospitalized surgical patients by medicine physicians in the United States. Arch Intern Med. 2010;170(4):363-368. doi:10.1001/archinternmed.2009.553

6. Huddleston JM, Long KH, Naessens JM, et al. Medical and surgical comanagement after elective hip and knee arthroplasty: a randomized, controlled trial. Ann Intern Med. 2004;141(1):28-38.

7. Tadros RO, Faries PL, Malik R, et al. The effect of a hospitalist comanagement service on vascular surgery inpatients. Journal of Vascular Surgery. 2015;61(6):1550-1555. doi:10.1016/j.jvs.2015.01.006

8. Hinami K, Feinglass J, Ferranti DE, Williams MV. Potential role of comanagement in "rescue" of surgical patients. Am J Manag Care. 2011;17(9):e333-339.

9. Rohatgi N, Loftus P, Grujic O, Cullen M, Hopkins J, Ahuja N. Surgical Comanagement by Hospitalists Improves Patient Outcomes: A Propensity Score Analysis. Ann Surg. 2016;264(2):275-282. doi:10.1097/SLA.0000000000001629

10. Auerbach AD, Wachter RM, Cheng HQ, et al. Comanagement of surgical 
patients between neurosurgeons and hospitalists. Arch Intern Med. 2010;170(22):2004-2010. doi:10.1001/archinternmed.2010.432 